\title{
Wüstenrot Stiftung (Hrsg.) (2020): Wohnen jenseits des Standards. Auf den Spuren neuer Wohnlösungen für ein differenziertes und bedürfnisgerechtes Wohnungsangebot
}

\author{
Thorsten Heitkamp
}

Eingegangen: 19. August 2021 - Angenommen: 14. September 2021 - Online veröffentlicht: 18. Oktober 2021

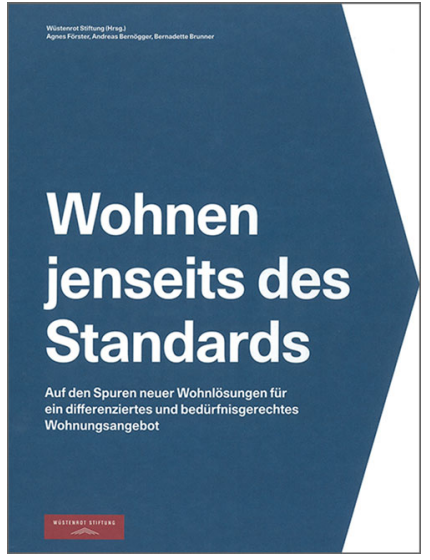

Die Publikation „Wohnen jenseits des Standards“, herausgegeben von der Wüstenrot Stiftung und verfasst von Agnes Förster, Andreas Bernögger und Bernadette Brunner von der Rheinisch-Westfälischen Technischen Hochschule Aachen, begibt sich, so der Untertitel, auf die „Spuren neuer Wohnlösungen für ein differenziertes und bedürfnisgerechtes Wohnungsangebot". Der zugrunde liegende Gedanke ist, dass sich zunehmend weiter ausdifferenzierende Le-

Dr. Thorsten Heitkamp, Liebigstraße 48, 44139 Dortmund, Deutschland

heitkamp@stadtforschung.de

(c) (1) () 2021 Heitkamp; licensee oekom verlag. This Open Access article is published under a Creative Commons Attribution 4.0 International License. bensstile neue (Wohn-)Bedürfnisse kreieren, die neuer Lösungen auf den Wohnungsmärkten im Bestand und Neubau unter der Vorgabe eines reduzierten Ressourceneinsatzes und -verbrauchs bedürfen. Neue Akteure, wie Genossenschaften, Baugruppen, Vereine und Stiftungen werden dabei als Pioniere gesehen, die ,Labore für innovative Lösungen ' anbieten, indem sie neue, gemeinschaftlich konzipierte und erschwingliche Wohnlösungen erforschen und bisherige Standards neu interpretieren. Dabei fasst das Buch die Erkenntnisse eines Forschungsprojektes der Wüstenrot Stiftung zu den „Möglichkeiten einer stärkeren Differenzierung des Wohnungsangebots" sehr strukturiert und grafisch gut aufbereitet mit dem Ziel zusammen, die vorgestellten Akteure und Projekte jenseits ihrer aktuellen Nische bekannter zu machen und Lernprozesse bei etablierten Marktakteuren anzustoßen.

Das Erkenntnisinteresse und Ziel des Forschungsprojekts stets im Blick behaltend, bietet die vorliegende Publikation einen fachlich holistischen Ansatz, der über das Gros der Betrachtungen neuer Wohnlösungen bzw. resilienter Quartiersentwicklungen (vgl. z. B. BBSR 2021; Schnur 2013), die oftmals eher auf immobilienwirtschaftliche und stadtentwicklungsplanerische Aspekte fokussieren, hinausgeht. Quartiere und Wohnlösungen „neu zu denken“, wie es vielerorts gefordert wird, erfordert jedoch mehr - und diesem Anspruch kommt die vorliegende Publikation erfreulich nahe.

Vier Kapitel gliedern die Publikation. Nach der Einleitung, die unter anderem das Erkenntnisinteresse, das Forschungsdesign und die Forschungsfragen darlegt, folgt, sozusagen als Grundlage der weiteren Analyse, ein gut strukturierter und logisch nachvollziehbarer Quervergleich von 
Rahmenbedingungen, Akteuren, Prozessen, Lösungen und Handlungslogiken aller 15 untersuchten Fallstudien. In einem folgenden Kapitel wird deren Übertragbarkeit analysiert. Diese drei Kapitel nehmen etwas mehr als die ersten 100 Seiten des Buches ein. Die weiteren der insgesamt 240 Seiten entfallen auf das vierte Kapitel mit insgesamt 15 Fallstudien zum „Wohnen jenseits des Standards“ mit jüngeren Wohnprojekten aus Deutschland, Österreich und der Schweiz, deren Auswahlkriterien und -strategie ebenfalls ausführlich und nachvollziehbar beschrieben werden. Der Anhang enthält unter anderem ein knappes Literaturverzeichnis.

Nach dem einführenden Kapitel startet das Kapitel 2 mit einem Quervergleich aller, wenig später in Kapitel 4 vorgestellten Projekte. Handlungsorientiert werden zunächst die Akteure in den Vordergrund gestellt: Wer sind sie, was bewegt sie, welche Kapazitäten bringen sie mit - aber etwa auch, welche Rolle Bauherren, Planer und Architekten im Kontext der Projekte einnehmen. Ähnlich detailliert werden die Entstehungs- und Entwicklungsprozesse der vorgestellten Projekte beschrieben, wobei ein Schwerpunkt der Analyse neben dem baulichen auf den sozialen Aspekt, aber auch auf die wichtige Phase der ,Inbetriebnahme“ des Projekts und die Organisation des Zusammenlebens im Projekt gelegt wird. Die gefundenen Lösungen werden detailliert aufgearbeitet und bieten einen äußerst interessanten Fundus an strukturiert dargelegten Erkenntnissen, die in vier verschiedene Themenbereiche unterteilt sind (Programm und Funktion, Kenngrößen, räumliche Struktur und Gestaltung, Organisation), die ihrerseits 24 weitere Themenfelder aufweisen. Ähnlich verhält es sich mit der glänzend aufgearbeiteten Analyse der Handlungslogiken der Akteure. Hier wird verständlich und nachvollziehbar, aus welch vielfältigen, sich oftmals überlagernden Gründen und mit welcher Grundmotivation die beteiligten Akteure sich auf das Abenteuer der Wohnprojekte ,jenseits des Standards“ einlassen.

Bei Projekten, so auch bei Wohnprojekten, stellt sich für Außenstehende die Frage nach ihrer Übertragbarkeit, auch wenn diese von den Projektakteuren vielleicht gar nicht intendiert ist. Innere Logiken von Projekten, die möglicherweise eine gewisse Allgemeingültigkeit aufweisen, können jedoch oftmals extrahiert werden und lassen grundlegende
Gemeinsamkeiten und Unterschiede sehr diverser Projekte erkennen. Auch das gelingt in der vorliegenden Publikation vorbildlich. Dieses wesentliche Kapitel der Publikation kommt ihrem angestrebten Ziel, Lernprozesse bei etablierten Marktakteuren anzustoßen, sehr nahe.

Insgesamt liegt eine ambitionierte und deutlich praxisorientierte Publikation vor, die aktuelle (Wohnungs-)Marktentwicklungen reflektiert und als Antwort auf die Rahmenbedingungen globaler Finanzmärkte, regionaler Wohnungsund lokaler Bodenmärkte und den durch ihnen insbesondere in den Kommunen ausgelösten Handlungsdruck evidenzbasiert neue Wohnlösungen detailliert und anregend vorstellt. Die Open-book-Version der Publikation ist der Printversion zumindest aus praktischen Gründen vorzuziehen, da im Text viele, durchaus hilfreiche Querverweise vorhanden sind, die eine Lektüre erleichtern. Alles in allem ist die vorliegende Publikation sorgfältig kuratiert. Sie bringt die interessanten Erkenntnisse des Wüstenrot-Forschungsprojekts allen Leserinnen und Lesern auf eine sehr anschauliche, gut lesbare und vor allen Dingen erkenntniserweiternde Weise näher. Die Relevanz der Publikation für die Praxis in den Kommunen und der Wohnungswirtschaft ist sehr hoch; aber auch Studierende können von der vorliegenden Publikation vielfältig profitieren.

\section{Vollständige bibliographische Angaben des rezensierten Werkes: \\ Wüstenrot Stiftung (Hrsg.) (2020): Wohnen jenseits des Standards. Auf den Spuren neuer Wohnlösungen für ein differenziertes und bedürfnisgerechtes Wohnungsange- bot. Ludwigsburg: Wüstenrot-Stiftung. 240 Seiten.}

\section{Literatur}

BBSR - Bundesinstitut für Bau-, Stadt- und Raumforschung (Hrsg.) (2021): Neue Stadtquartiere - Konzepte und gebaute Realität. Bonn. $=$ BBSR-Online-Publikation $04 / 2021$.

Schnur, O. (2013): Resiliente Quartiere? Eine Annäherung über das Panarchie-Modell adaptiver Zyklen. In: Informationen zur Raumentwicklung 4, 337-350. 